Pacific

Journal of

Mathematics

WIDTH COMPLEXES FOR KNOTS AND 3-MANIFOLDS

JENNIFER SCHULTENS

Volume 239 No. 1

January 2009 


\title{
WIDTH COMPLEXES FOR KNOTS AND 3-MANIFOLDS
}

\author{
JENNIFER SCHULTENS
}

\begin{abstract}
We define the width complexes for knots and 3-manifolds. We consider basic cases and reformulate the stabilization problem for Heegaard splittings from this point of view.
\end{abstract}

Simplicial complexes, cell complexes and more general complexes have featured prominently in geometric group theory for many years. Many such complexes have proved useful in the study of 3-manifolds. One example is the curve complex, introduced by W. Harvey [1979] and extensively studied by H. Masur and Y. Minsky [1999; 2000] as well as J. Hempel [2001]. We here define and study a rather different complex: the "width complex" for a knot or a 3-manifold. The construction grows out of a desire to better understand the workings of thin position, both in the context of knots and in the context of 3-manifolds. The notion of thin position for a knot was introduced in [Gabai 1987]. He used this notion to prove Property R for knots. It was also used by M. Culler, C. Gordon, J. Luecke and P. Shalen in their seminal work concerning Dehn surgery on knots [Culler et al. 1987]. The related notion of thin position for 3-manifolds was later introduced by M. Scharlemann and A. Thompson [1994]. It too has become a fundamental tool in the study of 3-manifolds.

Roughly speaking, in the case of knots, vertices of the width complex of a knot consist of appropriate equivalence classes of embeddings of the circle with respect to a height function, that is to say, a Morse function with exactly two critical points. In the case of 3-manifolds, they correspond to appropriate equivalence classes of Morse functions. In the case of knots, edges correspond to specified isotopies of embeddings. In the case of 3-manifolds, they correspond to the effect of passing through the singularities of Cerf theory. Higher dimensional simplices can be defined when edges correspond to independent alterations.

Research to date provides glimpses of the width complex. Many results can be interpreted or reformulated from this point of view. In effect, this highlights some of the formal similarities and differences between Heegaard splittings and certain aspects of knot theory. Most interestingly, it provides a reformulation of the stabilization problem for Heegaard splittings of 3-manifolds.

MSC2000: primary 57N10; secondary 57M25.

Keywords: width of knots, width of 3-manifolds, Cerf theory. 


\section{Definitions}

For standard definitions and results concerning knots, see [Burde and Zieschang 2003], [Lickorish 1997] or [Rolfsen 1990]. For standard definitions and results pertaining to 3-manifolds, see [Hempel 2004] or [Jaco 1980].

Definition 1. A height function on $\mathbb{S}^{3}$ is a Morse function with exactly two critical points.

This last assumption guarantees that $h$ induces a foliation of $S^{3}$ by spheres, along with one maximum that we denote by $\infty$ and one minimum that we denote by $-\infty$.

Definition 2. Let $K$ be a knot in $S^{3}$. If all minima of $\left.h\right|_{K}$ occur below all maxima of $\left.h\right|_{K}$, then we say that $K$ is in bridge position with respect to $h$. The index is the number of maxima (minima). The bridge number of $\mathrm{K}, b(K)$, is the minimal number of maxima required for $\left.h\right|_{K}$.

If $K$ is in bridge position, then a regular level surface below all maxima and above all minima is called a bridge sphere. Two bridge spheres are considered equivalent if their intersections with the complement of the knot are isotopic.

Definition 3. A compression body is a 3-manifold $W$ obtained from a closed orient-able surface $S$ by attaching 2-handles to $S \times\{0\} \subset S \times I$ and capping off any resulting 2 -sphere boundary components with 3-handles. We denote $S \times\{1\}$ by $\partial_{+} W$ and $\partial W \backslash \partial_{+} W$ by $\partial_{-} W$. Dually, a compression body is an orientable 3manifold obtained from a closed orientable surface $\partial_{-} W \times I$ or a $3-$ ball or a union of the two by attaching 1-handles.

In the case where $\partial_{-} W=\varnothing$, we also call $W$ a handlebody.

Definition 4. A Heegaard splitting of a 3-manifold $M$ is a pair $(V, W)$ in which $V$, $W$ are compression bodies and such that $M=V \cup W$ and $V \cap W=\partial_{+} V=\partial_{+} W=S$. We call $S$ the splitting surface or Heegaard surface. Two Heegaard splittings are considered equivalent if their splitting surfaces are isotopic.

The genus of $M$, denoted by $g(M)$, is the smallest possible genus of the splitting surface of a Heegaard splitting for $M$.

Definition 5. Let $(V, W)$ be a Heegaard splitting. A Heegaard splitting is stabilized if there is a pair of disks $(D, E)$ with $D \subset V$ and $E \subset W$ such that $\# \partial D \cap \partial E \mid=1$. We call the pair of disks $(D, E)$ a stabilizing pair of disks. A Heegaard splitting is unstabilized if it is not stabilized.

Definition 6. Destabilizing a Heegaard splitting $(V, W)$ is the act of creating a Heegaard splitting from $(V, W)$ by performing ambient 2-surgery on $S$ along the cocore of a 1-handle in either $V$ or $W$. 
In the above definition, the result of performing ambient 2-surgery on $S$ along the cocore of a 1-handle in either $V$ or $W$ is required to be a Heegaard splitting. This is not always possible! In order for the ambient 2-surgery to be a destabilization, the result is required to be a Heegaard splitting. This can be guaranteed if $(D, E)$ is a stabilizing pair of disks. In this case $D$ is the cocore of a 1-handle of $V$ and the existence of $E$ guarantees that the result of cutting along $D$ is a Heegaard splitting.

Definition 7. A tunnel system for a knot $K$ in $\mathbb{S}^{3}$ is a collection of $\operatorname{arcs} t_{1}, \ldots, t_{n}$ such that the complement of $K \cup t_{1} \cup \cdots \cup t_{n}$ is a handlebody. The tunnel number of a knot $K$ is the least number of arcs required for a tunnel system of $K$.

\section{The width complex for knots and 3-manifolds}

We wish to consider the possible positionings of a knot with respect to a height function. To do so, we define a complex. Roughly speaking, vertices of the width complex of a knot should consist of appropriate equivalence classes of embeddings of the given knot relative to a fixed height function. Width will be well-defined on vertices. Edges should correspond to isotopies that affect the width of the embedding. Our goal is to phrase the definition of the width complex for knots in concrete terms.

Analogously, we wish to consider the possible positionings of a 3-manifold, that is, the possible handle decompositions of the 3-manifold. This amounts to considering Morse functions on the 3-manifold. Again we define a complex. But here, rather than opting for the more concrete terminology used in the case of a knot, we will opt for the language of Morse functions and Cerf theory. This allows for a more abstract, but more concise, description of the width complex of a 3manifold.

2.1. The width complex for knots. We begin by fixing the height function $h$ : $\mathbb{S}^{3} \rightarrow \mathbb{R}$. Let $K$ be a knot in $\mathbb{S}^{3}$. Then $K=[k]$ is a smooth isotopy class of smooth embeddings of $\mathbb{S}^{1}$ into $\mathbb{S}^{3}$. We will denote a representative of $K$ by $k$ or by $k^{\prime}, k^{\prime \prime}, \ldots$, when we wish to distinguish between distinct representatives, i.e., embeddings. In the following discussion of the width complex for knots, the height function $h$ will be fixed once and for all. Note that the theory developed below could be developed by fixing $k$ and varying $h$. This would provide a clearer correspondence between our discussion of knots and our discussion of 3-manifolds. On the other hand, fixing $h$ allows for a more concrete discussion in the case at hand.

We first define the vertices of the width complex for a knot $K$. To this end, let $k$ be a representative of $K$ and let $c_{0}, \ldots, c_{n}$ be the critical values of $\left.h\right|_{k}$. Choose regular values $r_{1}, \ldots, r_{n}$ such that $c_{i-1}<r_{i}<c_{i}$. We call $R_{i}=h^{-1}\left(r_{i}\right)$ a thick 
level if $c_{i-1}$ corresponds to a local minimum and $c_{i}$ corresponds to a local maximum. We call $R_{i}=h^{-1}\left(r_{i}\right)$ a thin level if $c_{i-1}$ corresponds to a local maximum and $c_{i}$ corresponds to a local minimum. Denote the thick levels by $C_{0}, \ldots, C_{l}$ and the thin levels by $N_{1}, \ldots, N_{l}$. A representative $k$ of $K$ thus determines a set of level surfaces $C_{0}, N_{1}, C_{1}, \ldots, N_{l}, C_{l}$. Abusing notation slightly, we will also denote those portions of these level surfaces that lie in the complement of $k$ by $C_{0}, N_{1}, C_{1}, \ldots, N_{l}, C_{l}$, respectively. We consider two representatives of $K$ to be equivalent if their collections of thick and thin levels are isotopic. To each of the resulting equivalence classes, we associate a vertex.

Remark 8. The width of $k$ coincides with

$$
\frac{1}{2} \sum_{0}^{l}\left(\#\left|\partial C_{i}\right|\right)^{2}-\frac{1}{2} \sum_{1}^{l}\left(\#\left|\partial N_{i}\right|\right)^{2}
$$

See for instance [Howards et al. 2007]. In particular, width is well-defined on equivalence classes of representatives and hence on vertices.

We now define the edges of the width complex of a knot. To this end, consider a representative $k$ of $K$. An upper (lower) disk for $k$ at level $L_{i}$ is the following: A disk whose interior is disjoint from the thick and thin levels and whose boundary is partitioned into two subarcs, one coinciding with a subarc of $K$ containing exactly one maximum (minimum) and no other critical points and the other a subarc of $L_{i}$. A strict upper (lower) disk is an upper (lower) disk whose interior contains no critical points.

Suppose that there is a pair of disks, $(D, E)$, such that $D$ is a strict upper disk and $E$ is a strict lower disk for $k$ at the thick level $C_{i}$. Suppose further that this pair of disks is either disjoint, or meets in one point of $k$. Then the pair $(D, E)$ describes an isotopy that changes the embedding $k$ to an embedding $k^{\prime}$. Both $k$ and $k^{\prime}$ are representatives of $K$. We say that $k^{\prime}$ is obtained from $k$ by the pair $(D, E)$. We associate an edge to a pair of vertices if and only if a pair of representatives of the two equivalence classes corresponding to the vertices differ in this way.

Lemma 1. Let $D^{\prime}, D^{\prime \prime}$ be strict upper disks for $k$ at the thick level $C_{i}$ and let $E^{\prime}, E^{\prime \prime}$ be strict lower disks for $k$ at the thick level $C_{i}$. Suppose that $D^{\prime}$ and $E^{\prime}$ are disjoint and $D^{\prime \prime}$ and $E^{\prime \prime}$ are disjoint. The representatives $k^{\prime}$ of $K$, obtained from $k$ by the pair $\left(D^{\prime}, E^{\prime}\right)$, and $k^{\prime \prime}$ of $K$, obtained from $k$ by the pair $\left(D^{\prime \prime}, E^{\prime \prime}\right)$ are equivalent if and only if $D^{\prime}$ is isotopic to $D^{\prime \prime}$ via a level preserving isotopy and $E^{\prime}$ is isotopic to $E^{\prime \prime}$ via a level preserving isotopy.

Proof. If $D^{\prime}$ is isotopic to $D^{\prime \prime}$ via a level preserving isotopy and $E^{\prime}$ is isotopic to $E^{\prime \prime}$ via a level preserving isotopy, then this isotopy describes an isotopy of the thick and thin levels of the resulting presentations. Hence the representatives are equivalent. 


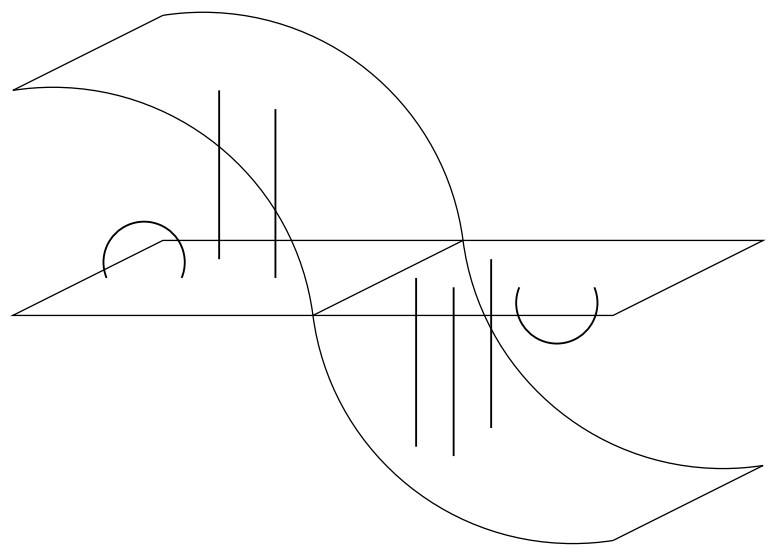

Figure 1. $N_{i}^{\prime}, N_{i}^{\prime \prime}, C_{i}$ and $k$.

Now suppose that $k^{\prime}$ and $k^{\prime \prime}$ are equivalent. By construction, $r=h\left(C_{i}\right)$ corresponds to a thin level $N_{i}^{\prime}=h^{-1}(r)$ for $k^{\prime}$ and a thin level $N_{i}^{\prime \prime}=h^{-1}(r)$ for $k^{\prime \prime}$. Then we can consider $N_{i}^{\prime}, N_{i}^{\prime \prime}$ and $C_{i}$ along with $k$. See Figure 1.

After a small isotopy, $N_{i}^{\prime}$ and $C_{i}$ meet in a single circle, as do $N_{i}^{\prime \prime}$ and $C_{i}$. Such a circle cuts each surface into two subdisks. We consider the 3-ball between a subdisk of $N_{i}^{\prime}$ and a subdisk of $C_{i}$. See Figure 2.

This 3-ball contains one subarc of $k$, call it $\alpha$, with both endpoints on the subdisk of $C_{i}$. All other subarcs of $k$ have one endpoint on the subdisk of $C_{i}$ and one subarc on the subdisk of $N_{i}^{\prime}$. The 3-ball admits only one disk (up to isotopy) cobounded by $\alpha$ and a subarc of $C_{i}$. Since $D^{\prime}$ is one such disk, this disk is isotopic to $D^{\prime}$. The same argument applies to $N_{i}^{\prime \prime}$ showing that $D^{\prime \prime}$ is isotopic to $D^{\prime}$.

Remark 9. The analogous result does not necessarily follow in the case that $D^{\prime}$ and $E^{\prime}$ and $D^{\prime \prime}$ and $E^{\prime \prime}$ meet in one point. Indeed, if there is exactly one maximum (the one on the boundary of $D^{\prime}$ ) above $C_{i}$ and below the next higher thin level and exactly one minimum (the one on the boundary of $E^{\prime}$ ) below $C_{i}$ and the next lower

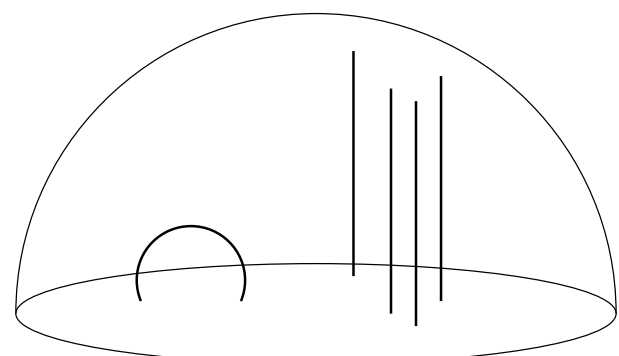

Figure 2. A 3-ball cobounded by a subdisk of $N_{i}^{\prime}$ and a subdisk of $C_{i}$. 

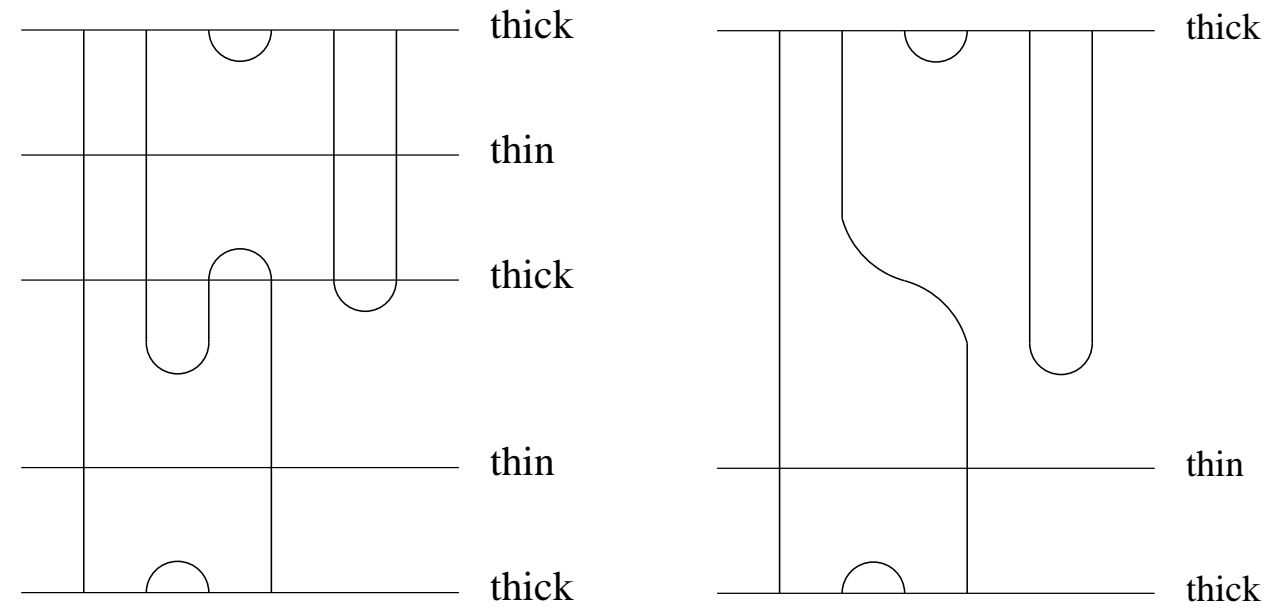

Figure 3. Left: Some thick and thin levels of $k$. Right: resulting thick and thin levels for $k^{\prime}$.

thin level, or if there are at least two maxima above $C_{i}$ and below the next higher thin level and at least two minima below $C_{i}$ and the next lower thin level, then the argument above establishes the analogous result. However, if these conditions are not met, then the conclusion need not follow. The obstruction in this latter case arises due to the fact that the new thick and thin levels are a subset of the former thick and thin levels and hence isotopic. See Figures 3 and 4.

Now consider the two pairs of disks $\left(D^{\prime}, E^{\prime}\right)$ and $\left(D^{\prime \prime}, E^{\prime \prime}\right)$ in Figure 5. The disks $E^{\prime}$ and $E^{\prime \prime}$ are not isotopic via a level preserving isotopy. If there were even more minima just below the thick level pictured in the two parts of Figure 5, then there would be even more strict lower disks not isotopic to $E^{\prime}$ via a level preserving isotopy.

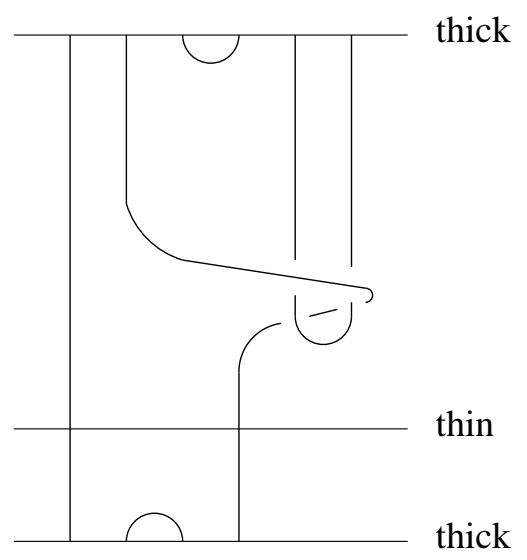

Figure 4. Some thick and thin levels for $k^{\prime \prime}$. 

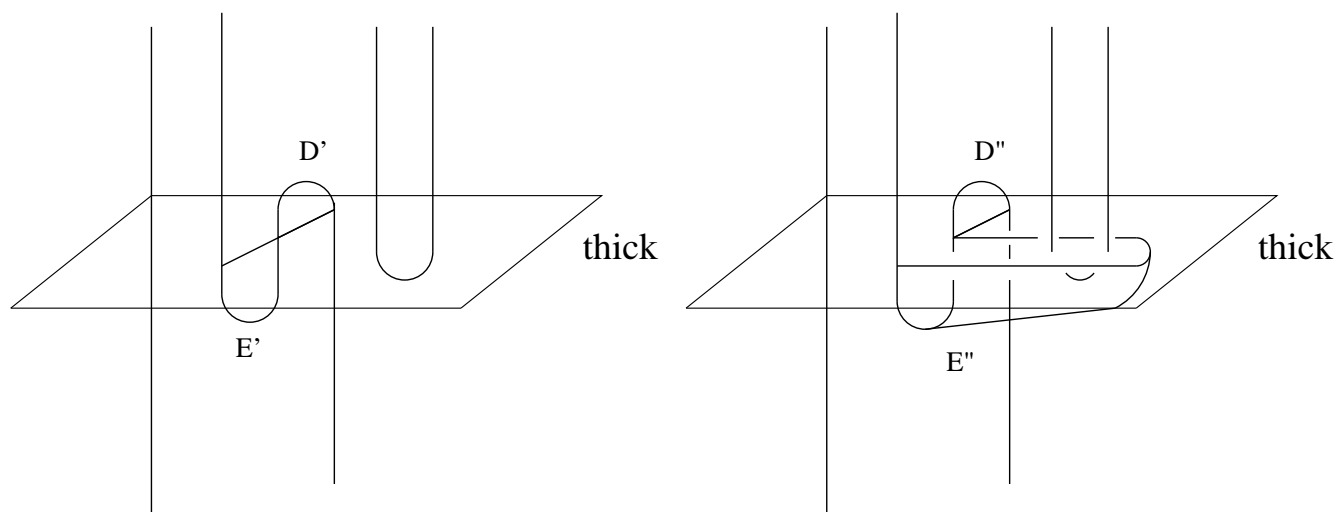

Figure 5. Left: A pair of disks yielding $k^{\prime}$. Right: A pair of disks yielding $k^{\prime \prime}$.

It follows from the above lemma that two vertices of the width complex can't be connected by more than one edge unless one of the vertices corresponds to a thick level exhibiting the obstruction described above.

We will refer to an isotopy described by a strict upper and strict lower disk that meet in a point of $K$ as a Type I move. We will refer to an isotopy described by a strict upper and strict lower disk that are disjoint as a Type II move. (This terminology is loosely inspired by the typology of Reidemeister moves.)

We now define higher dimensional cells of our complex: Suppose $k$ is a representative of $K$ and suppose that there are $n$ pairs of strict upper and strict lower disks at the thick levels where in each pair the two disks are either disjoint or meet in one point and such that distinct pairs are disjoint. Then these $\mathrm{n}$ pairs of disks describe $\mathrm{n}$ isotopies that can be performed in any order. This gives rise to $l$ other representatives (where $l \leq 2^{n}-1$ ). We associate an $l$-simplex to any $l$-tuple of vertices corresponding to an $l$-tuple of equivalence classes of representatives that differ in this way. (We think of these cells as $n$-dimensional cubes.)

By Remark 8 there is a function on the vertices of the width complex. This function can be extended continuously to give a combinatorial Morse function on the width complex. There are natural questions that can be asked about any complex. These pertain to connectivity properties and, more generally, the shape of the complex. The following theorem begins to address these issues.

\section{Theorem 1. The width complex of a knot is connected.}

Proof. Let $K$ be a knot in $\mathbb{S}^{3}$ and let $k, k^{\prime}$ be representatives of vertices $v, v^{\prime}$ of the width complex of $K$. Projecting $k, k^{\prime}$ onto a vertical plane does not change their respective equivalence classes. 
Recall that any two planar projections of a knot can be deformed into each other via a sequence of Reidemeister moves and planar isotopies. Planar isotopies either do not change the isotopy class of a representative or change it by Type I and Type II moves (corresponding to edges in the width complex of $K$ ). Likewise, a Reidemeister move either does not change the isotopy class of a representative or changes it by Type I and Type II moves (depending on the orientation of the Reidemeister move with respect to the projection of the height function on $\mathbb{S}^{3}$ to the vertical plane under consideration).

2.2. On the width complex of the unknot. We wish to illustrate the concept of the width complex of a knot. To this end we discuss the case of the unknot. As it turns out, the width complex of the unknot is already rather complicated. Nevertheless, some features are readily apparent. Theorem 2 below, due to J. P. Otal, establishes the uniqueness of bridge spheres for the unknot. It follows from this lemma that the width complex of the unknot contains an infinite ray with vertices $v_{1}, v_{2}, v_{3}, \ldots$, where $v_{n}$ corresponds to the bridge presentations of the unknot of index $n$. The edge between $v_{n}$ and $v_{n+1}$ corresponds to a Type I move.

Theorem 2. All bridge spheres of the unknot of a given index are equivalent.

This was first proved by J. P. Otal [1982]. We outline here an independent proof:

First consider two embeddings of the unknot in bridge position of the same index lying in a vertical plane. It is not hard to see that two such embeddings are isotopic through isotopies preserving the index. See Figure 6. This isotopy induces an appropriate isotopy of bridge spheres.

Now we must ascertain that any embedding of the unknot in bridge position can be isotoped to lie in a vertical plane while preserving the index. To this end, let $k$ be an embedding of the unknot in bridge position and let $D$ be a disk such that $\partial D=k$. We may assume that $\left.D\right|_{h}$ has as few critical points as possible in the interior of $D$. Then $D$ decomposes into polygons with an even number of edges
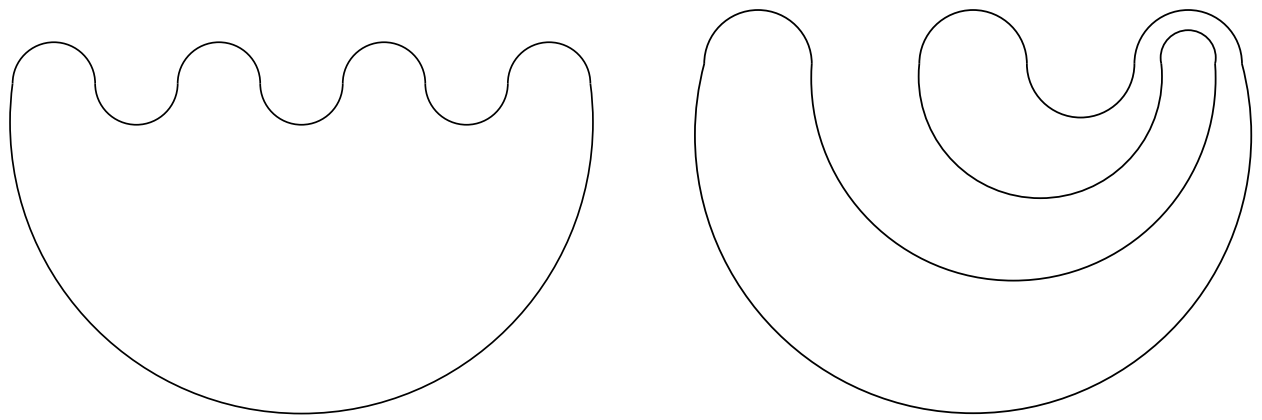

Figure 6. Two "planar" unknots of bridge index 4. 
such that these edges lie alternately in $K$ and in the bridge sphere. Each such polygon can be "flattened" to lie in a vertical plane. See Figures 7, 8 and 9.

We construct a graph $\Gamma$ as follows: To each polygon as above, we associate a vertex. We associate an edge to a pair of vertices if the corresponding polygons meet along an arc in the bridge sphere. Since each arc in the disk $D$ is separating, there can be no cycles in $\Gamma$. Thus $\Gamma$ is a tree (and can be called the "dual tree" to the decomposition of $D$ ).

The depth of a vertex is the least number of edges one must traverse to reach an endvertex. Beginning at a vertex $v$ of $\Gamma$ of maximal depth, we can isotope the polygon corresponding to $v$ to lie in a vertical plane $P$. Continuing with an adjacent vertex $v^{1}$, one edge of the polygon corresponding to $v^{1}$ already lies in $P$, but since we can isotope maxima and minima respectively, past maxima and minima respectively, we can isotope the rest of this polygon to lie in $P$. We continue in this fashion until we reach an endvertex $v^{n}$. At this stage, either $D$ has been

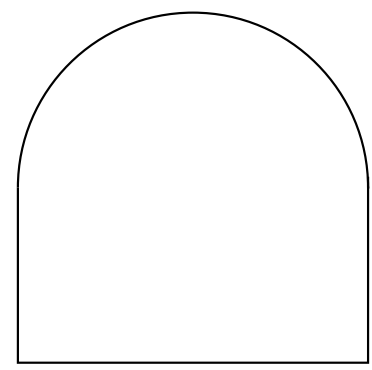

Figure 7. A flat bigon.
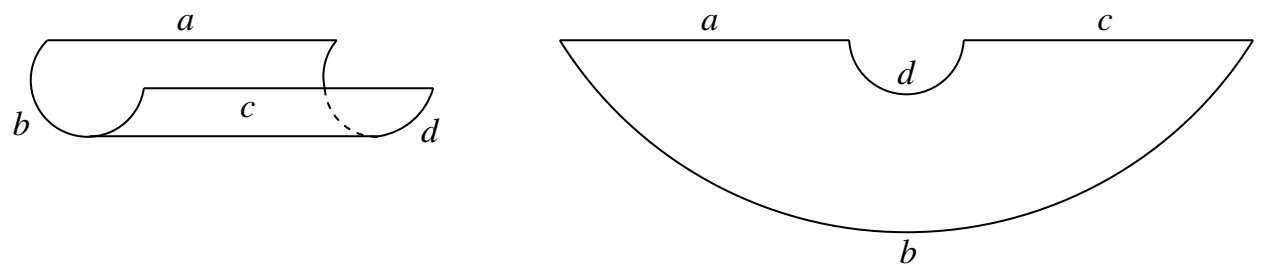

Figure 8. Flattening a quadrilateral.
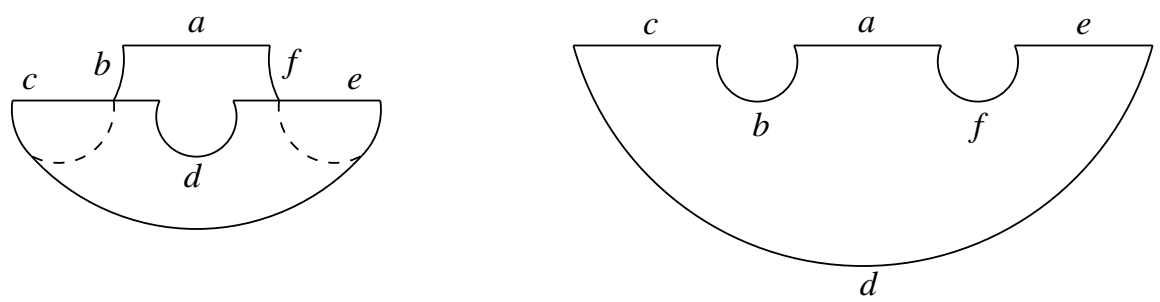

Figure 9. Flattening a hexagon. 


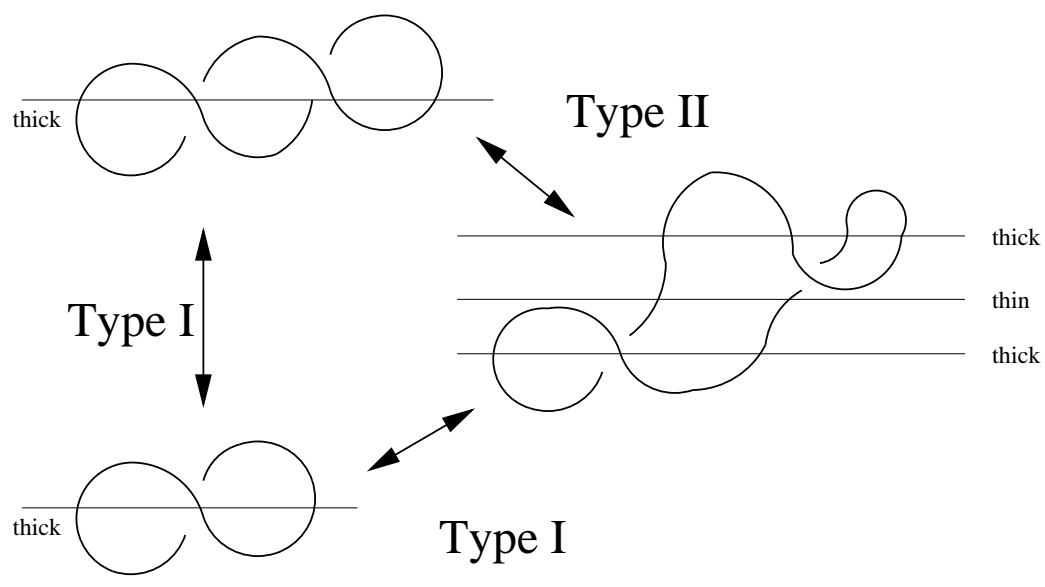

Figure 10. A cycle.

isotoped to lie entirely in $P$, or we continue with a vertex of maximal depth among $\Gamma \backslash\left\{v, v^{1}, \ldots, v^{n}\right\}$. At each stage, the polygon corresponding to the vertex $v^{k}$ under consideration has only one edge in $P$, so we can isotope it to lie in $P$.

Theorem 3. The width complex of the unknot contains an infinite ray.

Proof. This follows from Theorem 2.

More explicitly, the width complex of the unknot contains an infinite ray with vertices $v_{1}, v_{2}, v_{3}, \ldots$, where $v_{n}$ corresponds to the bridge presentation of the unknot of index $n$. Moreover, there is an edge between $v_{n}$ and $v_{n+1}$ corresponding to a Type I move. This describes the only edge emanating from $v_{1}$, the absolute minimum of the complex.

There are three edges emanating from $v_{2}$. One downwards, towards $v_{1}$, and one upwards, towards $v_{3}$. These two edges correspond to Type I moves. But there is another edge, towards a vertex that we denote by $v_{3}^{\prime}$. Here $v_{3}^{\prime}$ corresponds to a representative of the unknot with critical points, in ascending order, minimum, minimum, maximum, minimum, maximum, maximum. The edge between $v_{2}$ and $v_{3}^{\prime}$ also corresponds to a Type I move. The vertex $v_{3}^{\prime}$ is also connected to $v_{3}$ by an ascending edge corresponding to a Type II move. This describes a triangle in the width complex. This triangle does not bound a 2-simplex. See Figure 10.

Theorem 4. The width complex of a knot is not simply connected.

Proof. This follows immediately from the discussion above.

Note that $v_{3}^{\prime}$ is the only vertex that corresponds to a presentation of the unknot with critical points, in ascending order, minimum, minimum, maximum, minimum, maximum, maximum. This can be established via an argument similar to that in 

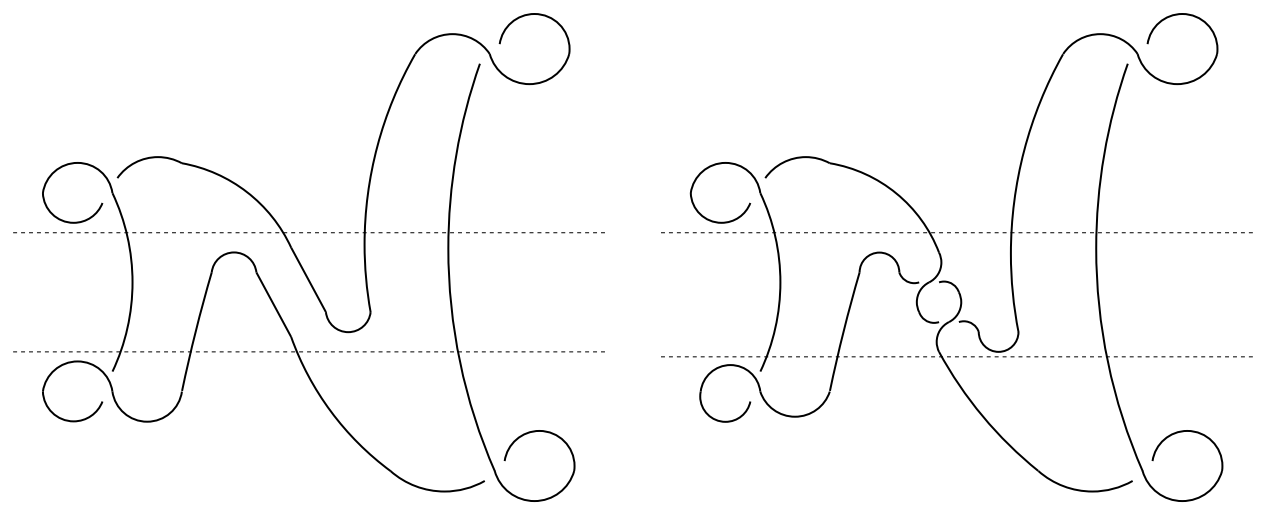

Figure 11. Two presentations of the unknot.

Theorem 2. (Here the thick level and the two thin levels decompose the disk $D$ bounded by $k$ into polygons. So we can again work with the dual tree. The only adjustment to the argument for Theorem 2 is that the flattening must commence by isotoping the single arc of intersection of $D$ with the thin level into $P$. Each of the polygons adjacent to this arc have maximal depth and we can proceed as in the argument for Theorem 2.)

Generically there are likely to be infinitely many vertices that correspond to presentations of the unknot with a given possible sequence of maxima and minima. The following Lemma hints at some of the possibilities:

Theorem 5. There exists a positive integer $N$, such that there are infinitely many distinct presentations of the unknot of width $N$.

Proof. If two presentations of the unknot are the same, then in particular, the corresponding portions of the knot complement cut out by adjacent thin levels must be homeomorphic. Now consider Figure 11, where the second and third thin levels are marked.

If the presentations coincide, then the portions of the knot complements cut out by the second and third thin levels are homeomorphic. They remain homeomorphic when we augment them by homeomorphic 3-manifolds. This implies that the two central components of the links pictured in Figure 12 have the same linking number.

But this is a contradiction. The same argument applies for any given number of twists between the two central components. Thus we exhibit an infinite family of presentations of the unknot with the same width.

Conjecture 1. Generically, there are infinitely many presentations of a given width for a given knot.

The example considered in the proof above provides us with an additional insight: 

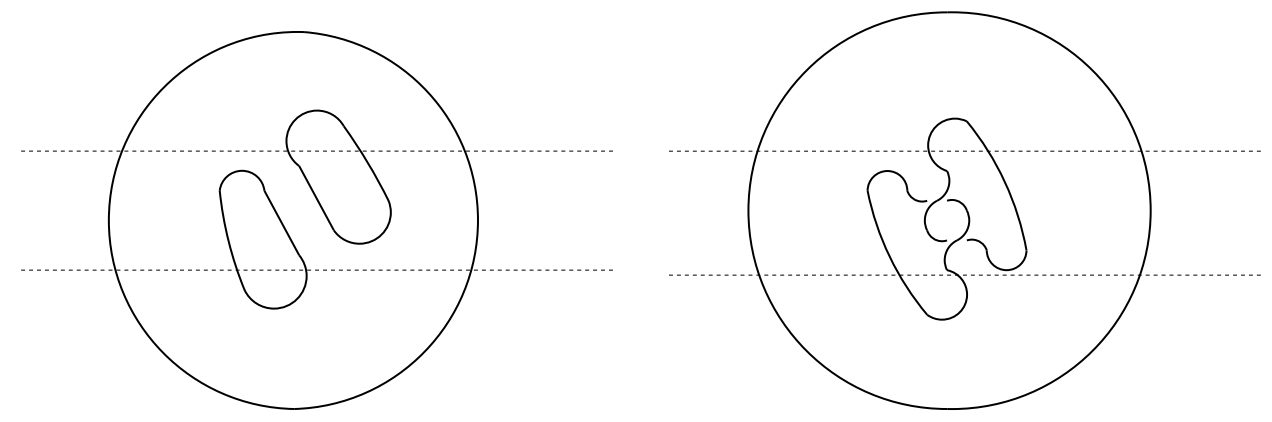

Figure 12. An augmentation and its analogue.

Theorem 6. The width complex of a knot is not locally finite.

Proof. Consider the infinitely many vertices $w_{0}, w_{ \pm 1}, w_{ \pm 2}, \ldots$ of the width complex of the unknot whose representatives are discussed in Theorem 5. In each such representative, raising all maxima to lie above all minima constitutes a sequence of Type II moves. In each case, this yields the bridge presentation of bridge index 7 . Thus in each case, the Type II moves correspond to a sequence of edges connecting the vertex $w_{i}$ to the vertex $v_{7}$ (in the infinite ray of Theorem 3). By the pigeon hole principle, there must be a path defined by such a sequence of edges, containing a vertex meeting infinitely many edges.

Problem 10. Is there a bound on the distance between vertices in terms of the width of the vertices?

2.3. The width complex for 3-manifolds. Let $M$ be a 3-manifold and consider the set of all real diffeomorphisms of $M$. The basic structure of this set was described by J. Cerf [1970]. The subset of Morse functions is open and dense in this set. Furthermore, the set contains codimension 1 strata consisting of those diffeomorphisms with either two nondegenerate critical points at the same level or one degenerate critical point of multiplicity 2. It contains codimension 2 strata consisting of those diffeomorphisms with either three nondegenerate critical points at the same level or one nondegenerate critical point and one degenerate critical point of multiplicity 2 at the same level or one degenerate critical point of multiplicity 3. Etc. Note that Morse functions are precisely those diffeomorphisms that do not lie in a codimension $\mathrm{n}$ stratum for $\mathrm{n}>0$.

We are interested in the dual complex of this stratified space, but after some modifications: We first absorb those codimension $\mathrm{n}$ strata consisting of diffeomorphisms with nondegenerate critical points of the same index at the same level into strata of lower codimension. We then absorb those codimension 1 strata corresponding to birth-death singularities involving either nondegenerate critical points 
of indices 0 and 1 or of indices 2 and 3 into the codimension 0 stratum and absorb strata of higher codimension involving such birth-death singularities into strata of lower codimension. The complex we wish to study is the dual complex of the remaining stratified space. Note that vertices of this space correspond to Morse functions and diffeomorphisms that fail to be Morse functions only because they have several nondegenerate critical points of the same index at the same level or because they exhibit birth-death singularities of the specified type.

Recall the notion of width for a Morse function introduced by M. Scharlemann and A. Thompson [1994]: For $M$ a 3-manifold and $h: M \rightarrow \mathbb{R}$ a Morse function we consider the critical values $c_{0}, \ldots, c_{n}$ of $h$ and choose regular values $r_{1}, \ldots, r_{n}$ such that $c_{i-1}<r_{i}<c_{i}$. For the purposes here we ignore critical values of index 0 or 3 and ignore inessential spheres occurring in the level surfaces considered below. We call $R_{i}=h^{-1}\left(r_{i}\right)$ a thick level if $c_{i-1}$ is a critical value of index 1 and $c_{i}$ is a critical value of index 2. We call $R_{i}=h^{-1}\left(r_{i}\right)$ a thin level if $c_{i-1}$ is a critical value of index 2 and $c_{i}$ is a critical value of index 1 . We denote the thick levels by $C_{0}, \ldots, C_{k}$ and the thin levels by $N_{1}, \ldots, N_{k}$. The width of $h$ is the $k$-tuple

$$
\#\left|C_{1}\right|-\chi\left(C_{1}\right), \ldots, \#\left|C_{k}\right|-\chi\left(C_{k}\right)
$$

but arranged in nonincreasing order.

Here for Morse functions corresponding to the same vertex the collections of thick and thin levels are isotopic. Thus the width of a vertex can be taken to be the width of a Morse function representing that vertex. This defines a function on the vertices of our complex. Since the function values on the vertices are $k$-tuples of numbers, with $k$ varying from vertex to vertex, this function does not extend to the complex. If we wish to define a function on the complex, we may do so by evaluating an appropriate algebraic expression on the $k$-tuples to obtain numbers associated with the vertices and extending these values to a function defined over the entire complex. (Here we will not be interested in the precise nature of such a function, though we will occasionally compare the widths of the vertices of the complex. This comparison is via the dictionary order.)

Consider the Morse function $h$ on $M$. A strict upper (lower) disk for a level $R$ is a disk whose boundary is an essential curve in $R$ and whose interior lies above (below) $R$ and is disjoint from all thick and thin levels. Consider a pair of disks for a thick level $C_{i}$ satisfying the following conditions: 1) One disk is a strict upper disk for $C_{i}$ and one disk is a strict lower disks for $C_{i}$;2) The boundaries of these two disks are either disjoint or meet in one point. Such a pair of disks can be used to define a modification of $h$ resulting in a new Morse function $h^{\prime}: M \rightarrow \mathbb{R}$. The strict upper disk corresponds to a 2-handle, the strict lower disk to a 1-handle. In the first case, the 2-handle can be attached before the 1-handle. This yields a new Morse function. In the second case, cutting along (either) one of the disks defines a 
new Morse function with two fewer critical points. In the language of Cerf theory, the first case corresponds to a critical point of index 1 and a critical point of index 2 passing each other and the second case corresponds to a birth-death singularity. Both events correspond to passing through a codimension 1 stratum in the stratified space considered above and vice versa. Hence these operations correspond to the edges in the width complex and vice versa.

Theorem 7. The width complex of a 3-manifold is connected.

Proof. This follows from standard results in Cerf theory.

2.4. The width complex of the 3-sphere. We wish to consider an example of the width complex of a 3-manifold. As it turns out, even the simplest of such examples, the width complex of $\mathbb{S}^{3}$, proves difficult. The structure of Heegaard splittings of $S^{3}$ provides a starting point for a description of this complex. Recall the following theorem:

Theorem 8. (Waldhausen) There is a unique minimal genus Heegaard splitting of $\mathbb{S}^{3}$ of genus 0 . Any other Heegaard splitting is obtained from this Heegaard splitting by stabilization. In particular, there is a unique Heegaard splitting of $S^{3}$ of any given genus.

It follows from Theorem 8 that the width complex of the 3 -sphere has one minimal width vertex corresponding to the Heegaard splitting of genus 0 and that it contains an infinite ray emanating from the minimal width vertex. Denote the vertices along this ray by $v_{0}, v_{1}, v_{2}, \ldots$ Here $v_{n}$ corresponds to the unique Heegaard splitting of $S^{3}$ of genus $n$. The edge between $v_{n}$ and $v_{n+1}$ corresponds to a stabilization.

There is only one edge meeting $v_{0}$, the edge contained in the ray mentioned above. When we consider stabilizations of Heegaard splittings, we think of the stabilization occurring at the unique thick level. In general, stabilizations can occur elsewhere as well. This is why there are three edges meeting $v_{1}$, the two edges contained in the above mentioned ray and an edge connecting $v_{1}$ to the vertex representing (all) Morse functions with critical points, taken in ascending order, of index 0 , index 1 , index 2 , index 1 , index 2 and index 3 . Denote this latter vertex by $v_{2}^{\prime}$. We can think of such a Morse function as obtained from a Morse function corresponding to the genus 1 Heegaard splitting of $S^{3}$ by stabilization either above or below all critical points. By Cerf's Triangle Lemma, the two cases yield Morse functions corresponding to the same vertex. For $v_{n}$ with $n>1$, there will be analogous edges and vertices.

There will be 5 edges meeting $v_{2}$. Two of these edges are contained in the above-mentioned ray and one edge, corresponding to a birth-death singularity, connects $v_{2}$ to $v_{2}^{\prime}$. In addition, there is one edge connecting $v_{2}$ to a vertex, call 


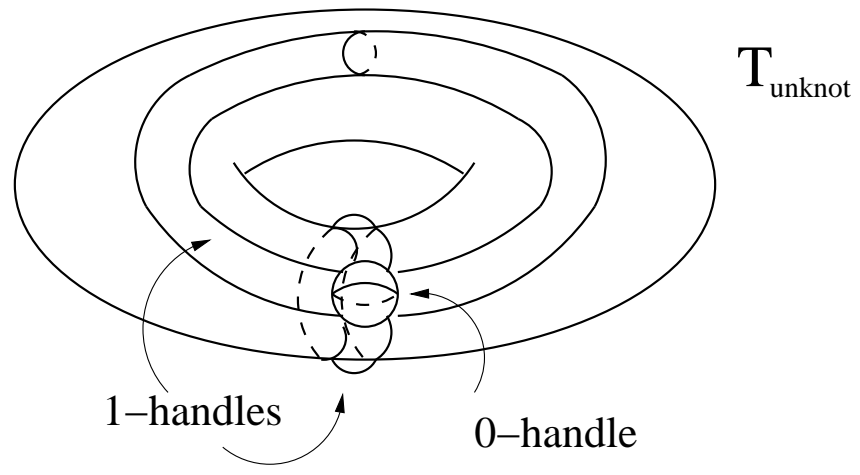

Figure 13. Sketch of the genus 2 handlebody for $T_{\text {unknot }}$.

it $v_{3}^{t}$, corresponding to a stabilization. Here the stabilization occurs above all index 1 and 2 critical points of the Morse function corresponding to $v_{2}$. If instead we stabilize between the highest and next highest critical point (here both these critical points are index 2), then by Cerf's Triangle Lemma, this yields a Morse function corresponding to $v_{3}^{t}$. Finally, there is one edge connecting $v_{2}$ to a vertex, call it $v_{3}^{b}$, corresponding to a stabilization below all critical points. If instead we stabilize between the lowest and next lowest critical point (here both critical points are index 1), then again, by Cerf's Triangle Lemma, this yields a Morse function corresponding to $v_{3}^{b}$. (Stabilizing above the two index 1 critical points and below the two critical points yields the vertex $v_{3}$ already mentioned.)

There will be infinitely many edges meeting $v_{3}$. This is because there are infinitely many distinct ways to move an index 1 critical point below an index 2 critical point. To see this, consider the following: Let $K \subset S^{3}$ be a knot of tunnel number 1. A closed regular neighborhood of this knot is a knotted solid torus $V_{K}$. Set $T_{K}=\partial V_{K}$. We can construct a manifold decomposition corresponding to a Morse function with critical points, in ascending order, of index 0 , index 1 , index 1 , index 2 , index 1 , index 2 , index 2 and index 3 that has $T_{K}$ as a thin level. Indeed, consider the genus 2 handlebody with handles whose cores run along the meridian and longitude of the knotted torus. This is $V_{1}$. See Figure 13 for a sketch of the genus 2 handlebody in the case that $K$ is the unknot.

Now construct a compression body $W_{1}$ with $\partial_{+} W_{1}=\partial_{+} V_{1}$ and $\partial_{-} W_{1}$ a torus parallel to $T_{K}$. Specifically, construct $W_{1}$ from $\partial_{+} W_{1} \times I$ by adding one 2-handle with cocore a shrunken copy of the disk bounded by the meridional 1-handle in $V_{1}$. In particular, $V_{1} \cup W_{1}=V_{K}$.

Now construct a compression body $V_{2}$ with $\partial_{-} V_{2}=\partial_{-} W_{1}$ and $\partial_{+} V_{2}$ a genus 2 surface. Specifically, construct $V_{2}$ from $\partial_{-} V_{2} \times I$ by adding a 1-handle with core a tunnel system realizing the tunnel number (it is 1 by assumption) for $K$. Finally, 
note that the remainder of $\mathbb{S}^{3}$ is a genus 2 handlebody that we denote by $W_{2}$. Here $\mathbb{S}^{3}=V_{1} \cup W_{1} \cup V_{2} \cup W_{2}$ is a generalized Heegaard splitting with thin level $T_{K}$.

Since there are infinitely many distinct tunnel number 1 knots, there are infinitely many knotted tori that can be used in this construction. The thin levels of the corresponding manifold decompositions aren't isotopic. Recall that for Morse functions corresponding to the same vertex, the collections of thick and thin levels must be isotopic. Thus the generalized Heegaard splittings described correspond to infinitely many Morse functions that in turn correspond to infinitely many distinct vertices $v_{3}^{1}, v_{3}^{2}, v_{3}^{3}, \ldots$. Since these Morse functions are obtained from the one corresponding to the genus 3 Heegaard splitting of $S^{3}$ by letting a critical point of index 1 pass a critical point of index 2 , there must be infinitely many edges, one between $v_{3}$ and $v_{3}^{1}$, one between $v_{3}$ and $v_{3}^{2}$, etc.

Theorem 9. There can be infinitely many vertices that have the same width in the width complex of a 3-manifold.

Proof. This follows immediately from the discussion above.

Theorem 10. The width complex of a 3-manifold is not locally finite.

Proof. The discussion above establishes the fact that the width complex of $\mathbb{S}^{3}$ is not locally finite. For any other 3-manifold $M$, appropriate connect sums of $M$, endowed with a Morse function, and $\mathbb{S}^{3}$, with each of the Morse functions constructed in the discussion above, yield the same phenomenon.

\section{Minima}

Traditional questions concerning minimal width embeddings of knots or of thin 3-manifold decompositions can be rephrased in terms of width complexes. For instance, a knot in thin position is represented by a vertex that is an absolute minimum for the width function on the width complex of the knot. Analogously, a thin manifold decomposition corresponds to a minimum width vertex in the width complex of a 3-manifold.

Any knot diagram can be changed to one of greater width via a Type I move. Thus the width complex of a knot contains no maxima. Analogously, any Morse function of a 3-manifold can be changed to one of greater width via stabilization of the corresponding handle decomposition. Thus the width complex of a 3-manifold contains no maxima.

As noted above, the width complex of the unknot contains a unique absolute minimum, represented by the round circle. The width complex of the 3-sphere contains a unique absolute minimum represented by the genus 0 Heegaard splitting. In general, one should not expect this sort of uniqueness. 
Question 11. Are there knots with width complexes containing distinct global minima?

Such knots are likely to exist. For instance, if a knot has distinct bridge spheres of the same index, and bridge position happens to be thin position for this knot, then the answer to this question is "yes". Also, any knot that has distinct thin positions provides an affirmative answer to this question. At this time, we believe that such knots exist, but do not know of any examples.

Theorem 11. There are 3-manifolds with width complexes that contain distinct global minima.

Proof. In [Boileau et al. 1989], the authors exhibit the distinct Heegaard splittings for small Seifert fibered spaces. Generically, these Heegaard splittings are strongly irreducible. Thus they represent distinct minima in the width complex of these manifolds.

In fact, we can say more:

Theorem 12. There are 3-manifolds with width complexes that contain infinitely many distinct global minima.

Proof. The 3-torus has a Heegaard splitting that is obtained by letting $V$ be a closed regular neighborhood of the 1-skeleton of a cubulation and letting $W$ be the closure of its complement. See [Boileau and Otal 1990]. This Heegaard splitting achieves the minimal genus of the 3-torus. The corresponding handle decomposition can be rearranged in infinitely many ways. The resulting handle decompositions will all be built of a 0-handle followed by two 1-handles, followed by one 2-handle, followed by one 1-handle, followed by two 2-handles, followed by one 3-handle. Each of these infinitely many handle decompositions has a unique thin level. This thin level will consist of two parallel incompressible tori. But the rearrangement of the handles can be conducted so as to realize any of the infinitely many isotopy classes of incompressible tori as the thin level. See Figures 14 and 15.

The handle decomposition depicted in Figure 14 is obtained by attaching one 0handle, two horizontal 1-handles, $a, b$, and a horizontal 2-handle before attaching a vertical 1-handle, $c$, two vertical 2-handles and a 3-handle. The thin level consists of two parallel horizontal tori.

The handle decomposition depicted in Figure 15 is obtained by attaching a horizontal 1-handle $a^{\prime}$, another 1-handle, $b^{\prime}$ (that runs from the vertex through the front right face of the cube, returns through the back left face of the cube, runs through the top face and returns to the vertex through the bottom face), then a slanted 2handle before attaching a vertical 1-handle, $c$, then two 2-handles and a 3-handle. The thin level consists of two parallel tori that intersect the front face of the cube at an angle of $\pi / 4$. 


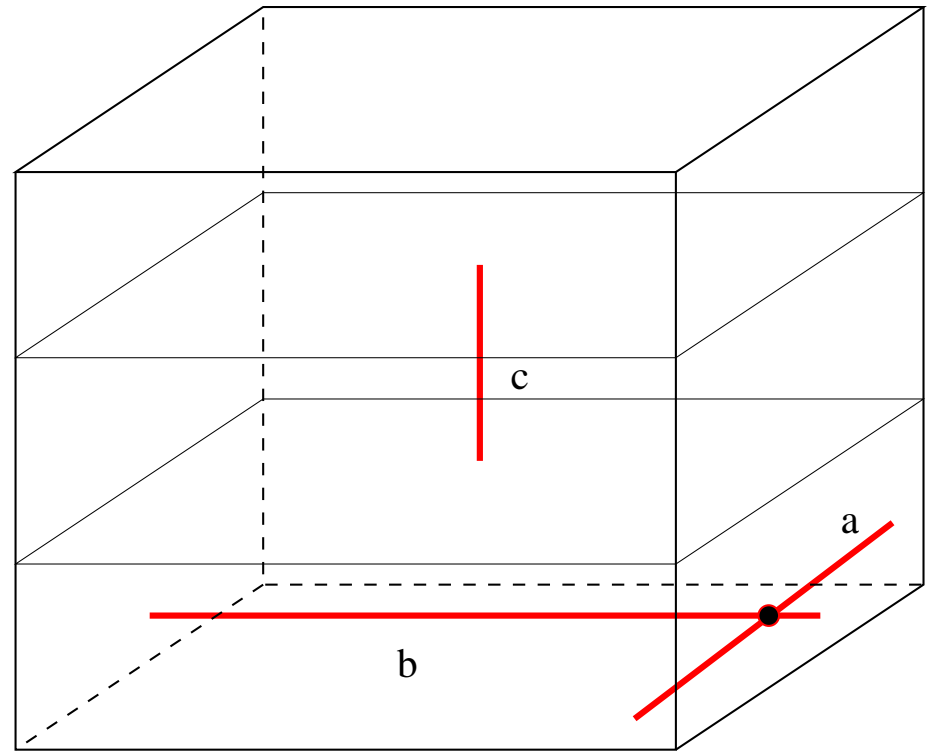

Figure 14. A handle decomposition of the 3-torus: the vertex represents a 0 -handle, the edges $a, b, c$ represent three 1 -handles, $c$ is attached to the two components of the thin level.

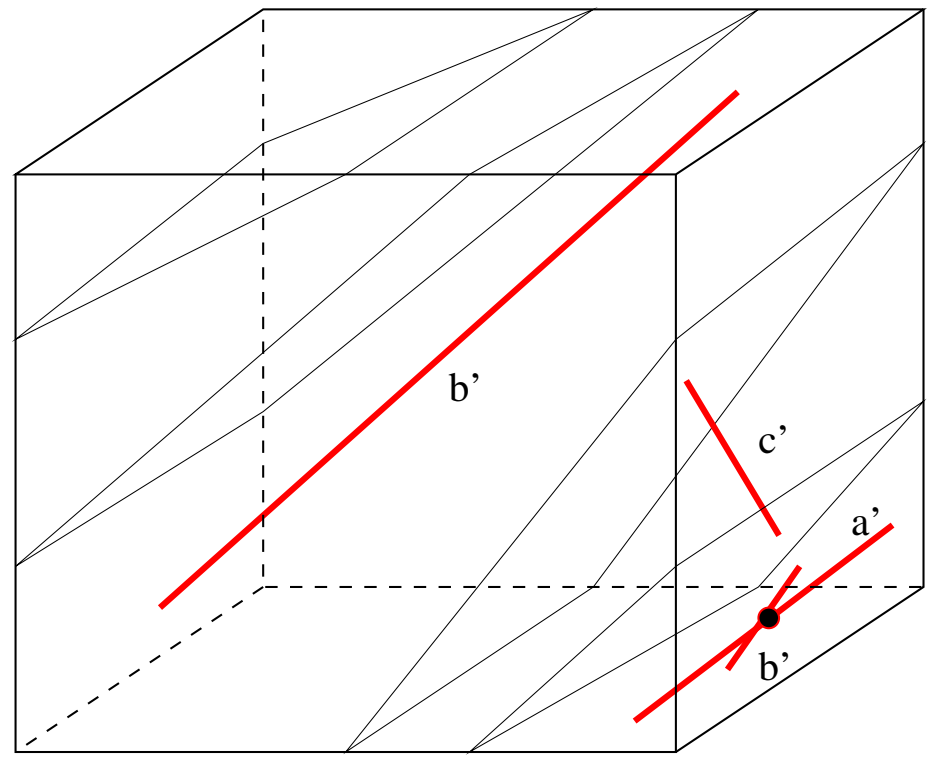

Figure 15. Another such handle decomposition: the vertex represents a 0 -handle, the edges $a^{\prime}, b^{\prime}, c^{\prime}$ represent three 1-handles, $c^{\prime}$ is attached to the two components of the thin level. 
Question 12. Can the width complex of a knot have local minima that are not global minima?

Theorem 13. The width complex of a 3-manifold can have local minima that aren't global minima.

Proof. A. Casson and C. Gordon [1986] have exhibited 3-manifolds that possess strongly irreducible Heegaard splittings of arbitrarily high genus. (See also [Kobayashi 1990; 1992].) Such Heegaard splittings correspond to local minima in the width complexes of these 3-manifolds. Thus there will be infinitely many local minima that are not global minima.

\section{Distances}

Earlier, in our discussion of the width complex of a knot, we asked whether or not there is a bound on the distance between vertices in terms of the width of the vertices. The analogous question can be asked in the context of the width complex of a 3-manifold. Answers to these questions provide information not only on distances, but on minima: For suppose that a local minimum is not a global minimum. Then the width of the global minimum is strictly less than, and hence bounded above by, the width of the local minimum. Thus a bound on the distance between the vertices in terms of the width of the vertices provides information on the distance of a local minimum from a global minimum.

4.1. Distance in the width complex of a knot. Questions pertaining to distance in the width complex of a knot are reminiscent of, but quite distinct from, questions about the computational complexity of Reidemeister moves. For instance, in 1934 Goeritz exhibited diagrams of the unknot with the property that any sequence of Reidemeister moves converting the diagram to the round circle would have to involve diagrams of the unknot with a larger number of crossings. In this vein one may ask the following:

Question 13. Is every vertex of the width complex of a knot connected to one of the global minima of this complex by a monotonically decreasing path?

A global minimum of the width complex is represented by a knot in thin position. This embedding can be transformed into an embedding in bridge position by isotoping all maxima to lie above all minima. In terms of the width complex, this means that there is a monotonically increasing path, consisting of type II edges, from the global minimum to a vertex represented by an embedding in bridge position. In fact, such a path exists for any vertex.

We will see below that the analogous idea proves fruitful with regards to the width complex of 3-manifolds. Here, this idea merely conjures up more questions: What can we say about the paths between the vertices represented by embeddings 
in bridge position? If there is a vertex from which there is no monotonically decreasing path to a global minimum, must any path from this vertex to a global minimum pass through a vertex represented by an embedding in bridge position? If the answer is "no", does there at least exist such a path of minimum length?

4.2. Distance in the width complex of a 3-manifold. One of the longstanding questions in the study of 3-manifolds concerns stabilization of Heegaard splittings.

Theorem 14 (Reidemeister-Singer). Any two Heegaard splittings of a 3-manifold are stably equivalent.

In the language here, the Reidemeister-Singer Theorem tells us that two vertices in the width complex of a 3-manifold corresponding to Heegaard splittings are connected by a path that consists of an ascending sequence of edges followed by a descending sequence of edges. This realization prompted the next natural question: Given two Heegaard splittings, what is the smallest possible genus of a common stabilization?

Definition 14. The smallest possible genus of a common stabilization of two Heegaard splittings is called the stable genus of the pair.

Theorem 15 (Rubinstein-Scharlemann). There is a linear function in two variables that provides an upper bound on the stable genus of pairs of Heegaard splittings of non-Haken manifolds. There is a quadratic function in two variables that provides an upper bound on the stable genus of pairs of Heegaard splittings of Haken manifolds.

This theorem tells us that in the width complex of a 3-manifold, a pair of vertices represented by Heegaard splittings is connected by a path with a single maximum. Moreover, the length of the path is bounded in terms of a function on the widths of the vertices (i.e., genera of the Heegaard splittings). In the context here, we are more interested in the distance between the vertices. These results do give us such a bound. Specifically, given any two vertices in the width complex of a 3-manifold, they are connected by ascending edges (the number of which is bounded by the width of these vertices) to vertices represented by Heegaard splittings. The latter, in turn, are connected by a path with length bounded by the function provided in the Rubinstein-Scharlemann Theorem.

One remaining question is whether or not the shortest path between two vertices can or must have exactly one maximum. Indeed, for vertices corresponding to Heegaard splittings such a path can always be chosen to have exactly one maximum. For consider a path $e_{1}, \ldots, e_{n}$ between such vertices. If a descending edge $e_{i}$ corresponding to a critical point of index 1 passing a critical point of index 2 is followed by an edge $e_{i+1}$ corresponding to a birth-death vertex, then there is an alternate path in which the order of these two edges is reversed. In other words, 


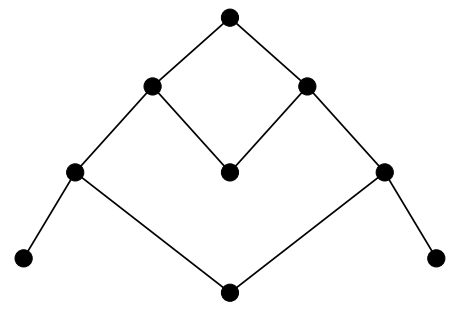

Figure 16. A subgraph of the width complex of a 3-manifold?

edges corresponding to birth-death vertices can always be assumed to lie above edges corresponding to a critical point of index 1 passing a critical point of index 2. Furthermore, though there can be infinitely many rearrangements of a handle decomposition corresponding to a Heegaard splitting, there is only one Heegaard splitting corresponding to a handle decomposition and all its rearrangements. The fact that the shortest path between two vertices corresponding to Heegaard splittings can always be chosen to have exactly one maximum hence follows from the fact that stabilization is well-defined, i.e., unique.

The analogous statement may or may not be true in general. Indeed, suppose two vertices correspond to distinct rearrangements of a specific handle decomposition. It appears possible that the width complex of a 3-manifold could have a subgraph as in Figure 16. All edges in this subgraph are meant to correspond to a critical point of index 1 passing a critical point of index 2. The shortest path from the lower left vertex to the lower right vertex then has length 4 and two maxima.

\section{Acknowledgments}

I wish to thank the Max-Planck-Institut für Mathematik in Bonn, Germany where this work was begun and the Max-Planck-Institut für Mathematik in den Naturwissenschaften in Leipzig, Germany, where this work was completed. The work was supported in part by a grant from the NSF.

\section{References}

[Boileau and Otal 1990] M. Boileau and J.-P. Otal, "Sur les scindements de Heegaard du tore $T^{3 \text { ", }}$ J. Differential Geom. 32:1 (1990), 209-233. MR 91i:57006 Zbl 0754.53012

[Boileau et al. 1989] M. Boileau, D. Collins, and H. Zieschang, "Scindements de Heegaard des petites variétes de Seifert”, C. R. Acad. Sci. Paris Sér. I Math. 305:12 (1989), 557-560. MR 88k:57003 Zbl 0651.57010

[Burde and Zieschang 2003] G. Burde and H. Zieschang, Knots, 2nd ed., Studies in Mathematics 5, de Gruyter, Berlin, 2003. MR 2003m:57005 Zbl 1009.57003

[Casson and Gordon 1986] A. Casson and C. Gordon, "Manifolds with irreducible Heegaard splittings of arbitrarily high genus", preprint, 1986. 
[Cerf 1970] J. Cerf, "La stratification naturelle des espaces de fonction différentiables réelles et le théorème de la pseudo-isotopie", Inst. Hautes Études Sci. Publ. Math. 39 (1970), 5-173. MR 45 \#1176 Zbl 0213.25202

[Culler et al. 1987] M. Culler, C. M. Gordon, J. Luecke, and P. B. Shalen, "Dehn surgery on knots", Ann. of Math. (2) 125:2 (1987), 237-300. MR 88a:57026 Zbl 0633.57006

[Gabai 1987] D. Gabai, "Foliations and the topology of 3-manifolds, III", J. Differential Geom. 26:3 (1987), 479-536. MR 89a:57014b Zbl 0639.57008

[Harvey 1979] W. J. Harvey, "Geometric structure of surface mapping class groups", pp. 255-269 in Homological group theory (Durham, 1977), edited by C. T. C. Wall, London Math. Soc. Lecture Note Ser. 36, Cambridge Univ. Press, Cambridge, 1979. MR 82h:57012 Zbl 0424.57006

[Hempel 2001] J. Hempel, "3-manifolds as viewed from the curve complex", Topology 40:3 (2001), 631-657. MR 2002f:57044 Zbl 0985.57014

[Hempel 2004] J. Hempel, 3-manifolds, AMS Chelsea Publishing, Providence, RI, 2004. Reprint of the 1976 original. MR 2005e:57053 Zbl 1058.57001

[Howards et al. 2007] H. Howards, Y. Rieck, and J. Schultens, "Thin position for knots and 3manifolds: A unified approach", Geom. Topol. Monographs 12 (2007), 89-120.

[Jaco 1980] W. Jaco, Lectures on three-manifold topology, CBMS Regional Conference Series in Mathematics 43, American Math. Soc., Providence, 1980. MR 81k:57009 Zbl 0433.57001

[Kobayashi 1990] T. Kobayashi, "There exist 3-manifolds with arbitrarily high genus irreducible Heegaard splittings", 1990.

[Kobayashi 1992] T. Kobayashi, "A construction of 3-manifolds whose homeomorphism classes of Heegaard splittings have polynomial growth", Osaka J. Math. 29:4 (1992), 658-674. MR 93j:57007 Zbl 0785.57005

[Lickorish 1997] W. B. R. Lickorish, An introduction to knot theory, Graduate Texts in Mathematics 175, Springer, New York, 1997. MR 98f:57015 Zbl 0886.57001

[Masur and Minsky 1999] H. A. Masur and Y. N. Minsky, "Geometry of the complex of curves, I: Hyperbolicity", Invent. Math. 138:1 (1999), 103-149. MR 2000i:57027 Zbl 0941.32012

[Masur and Minsky 2000] H. A. Masur and Y. N. Minsky, "Geometry of the complex of curves, II: Hierarchical structure”, Geom. Funct. Anal. 10 (2000), 902-974. MR 2001k:57020 Zbl 0972.32011

[Otal 1982] J. Otal, "Présentations en ponts du nœud trivial", C. R. Acad. Sci. Paris Sér. I Math. 294:16 (1982), 553-556. MR 84a:57006 Zbl 0498.57001

[Rolfsen 1990] D. Rolfsen, Knots and links, Mathematics Lecture Series 7, Publish or Perish, Houston, TX, 1990. Corrected reprint of the 1976 original. MR 95c:57018 Zbl 0854.57002

[Scharlemann and Thompson 1994] M. Scharlemann and A. Thompson, "Thin position for 3-manifolds”, pp. 231-238 in Geometric topology (Haifa, 1992), edited by C. Gordon et al., Contemp. Math. 164, Amer. Math. Soc., Providence, RI, 1994. MR 95e:57032 Zbl 0820.57005

Received January 28, 2008. Revised July 30, 2008.

\author{
JENNIFER SCHULTENS \\ DEPARTMENT OF MATHEMATICS \\ 1 Shields AVEnue \\ UNIVERSITY OF CALIFORNIA \\ DAVIS, CA 95616 \\ UNITED STATES \\ jcs_business@hotmail.com
}

